\title{
Mortality from cancer (other than lung) and radon exposure in German uranium miners, 1946-2013
}

\author{
Nora Fenske $^{1, *}$, Veronika Deffner ${ }^{1}$, Maria Schnelzer $^{1}$, Christina Sobotzki ${ }^{1}$, and Michaela \\ Kreuzer $^{1}$ \\ ${ }^{1}$ Federal Office for Radiation Protection (BfS), Division Effects and Risks of Ionising and Non- \\ Ionising Radiation, Neuherberg, Germany
}

\section{Background}

Radon is an established risk factor for lung cancer. Less clear is whether radon can cause cancers other than lung cancer. Due to the several to many times lower organ doses for organs other than the lung, the radon-related risks - if any - are expected to be much lower than the effect of radon on lung cancer. Therefore, large study populations with a wide range of exposures and long observation periods are required to investigate and to provide evidence for such associations. Based on the current follow-up period (1946-2013) of the German uranium miner cohort study $(\mathrm{n}=58,974$ men) the association between mortality from cancers other than lung cancer and cumulative exposure to radon and its progeny (mean=280, median=33, $\max =3224$ WLM) was investigated.

\section{Methods}

Annual exposures to radon and its progeny in working level months (WLM) and to external gamma radiation in $\mathrm{mSv}$ were retrospectively assessed using a comprehensive job-exposure matrix. Considered endpoints were the group of non-lung cancers as well as all individual cancer sites with more than 35 deaths. Standardized mortality ratios were calculated to compare cancer mortality rates observed in the cohort with those of the general male population in Eastern Germany. Statistical modelling of the mortality rates in the cohort was performed by internal Poisson regression for grouped data with baseline stratification by age and calendar year. Excess relative risks (ERR) per $100 \mathrm{WLM}$ and $95 \%$ confidence intervals $(\mathrm{CI})$ were estimated for cumulative 5-year lagged exposure to radon. Numerous additional and sensitivity analyses were conducted, such as additive adjustment for cumulative exposure to external gamma radiation, modelling of non-linear associations, modelling of effect modifications by time-dependent factors, or additional stratification for workplace and duration of employment.

* Corresponding author: nfenske@bfs.de 


\section{Results}

A statistically significant association was found for the group of non-lung cancers $(\mathrm{n}=5,122 ; \mathrm{ERR} / 100 \mathrm{WLM}=0.014 ; 95 \% \mathrm{CI}: 0.006,0.021)$ which did not change after adjustment for external gamma radiation. Additional analyses indicated an effect modification depending on age at exposure. With respect to individual cancer sites, the majority of risk estimates were positive (14 out of 18), and two of them reached borderline significance (colon and liver cancer), even after adjustment for external gamma radiation. The largest ERR coefficients were estimated for pharynx, liver and non-Hodgkin lymphoma.

Overall, the results are in line with those based on previous follow-up periods [1-5], but the increased statistical power leads to smaller confidence intervals for estimated ERR coefficients (in comparable statistical analyses [1,2]).

\section{Conclusion}

The present analysis provides some evidence of an increased risk for cancers other than lung cancer associated with radon, but confounding cannot be ruled out. Limiting factors of the analysis are the lack of data on cancer incidence, lack of information on potential confounders (such as alcohol consumption and smoking), and the uncertainty of exposure in early years. Several projects are ongoing to address these drawbacks: an international pooling of uranium miner studies (PUMA), a linkage with the German cancer registry, and uncertainty analyses in the German uranium miner cohort.

\section{References}

1. M. Kreuzer, L. Walsh, M. Schnelzer, A. Tschense, B. Grosche, Br. J. Cancer 99 (2008)

2. M. Kreuzer, B. Grosche, M. Schnelzer, A. Tschense, F. Dufey, L. Walsh, Radiat. Environ. Biophys. 49 (2010)

3. M. Kreuzer, K. Straif, J.W. Marsh, F. Dufey, B. Grosche, D. Nosske, M. Sogl, Occup. Environ. Med. 69 (2012)

4. F. Dufey, L. Walsh, M. Sogl, A. Tschense, M. Schnelzer, M. Kreuzer, J. Radiol. Prot. 33 (2013)

5. M. Kreuzer, F. Dufey, J.W. Marsh, D. Nowak, M. Schnelzer, L. Walsh, Int. J. Rad. Biol. (2014) 